\title{
Ankylosing Spondylitis
}

\section{Anterior Spondylitis}

- A 54-year-old male

- Chronic dorsal and lumbar pain

- Presence of HLA-B27
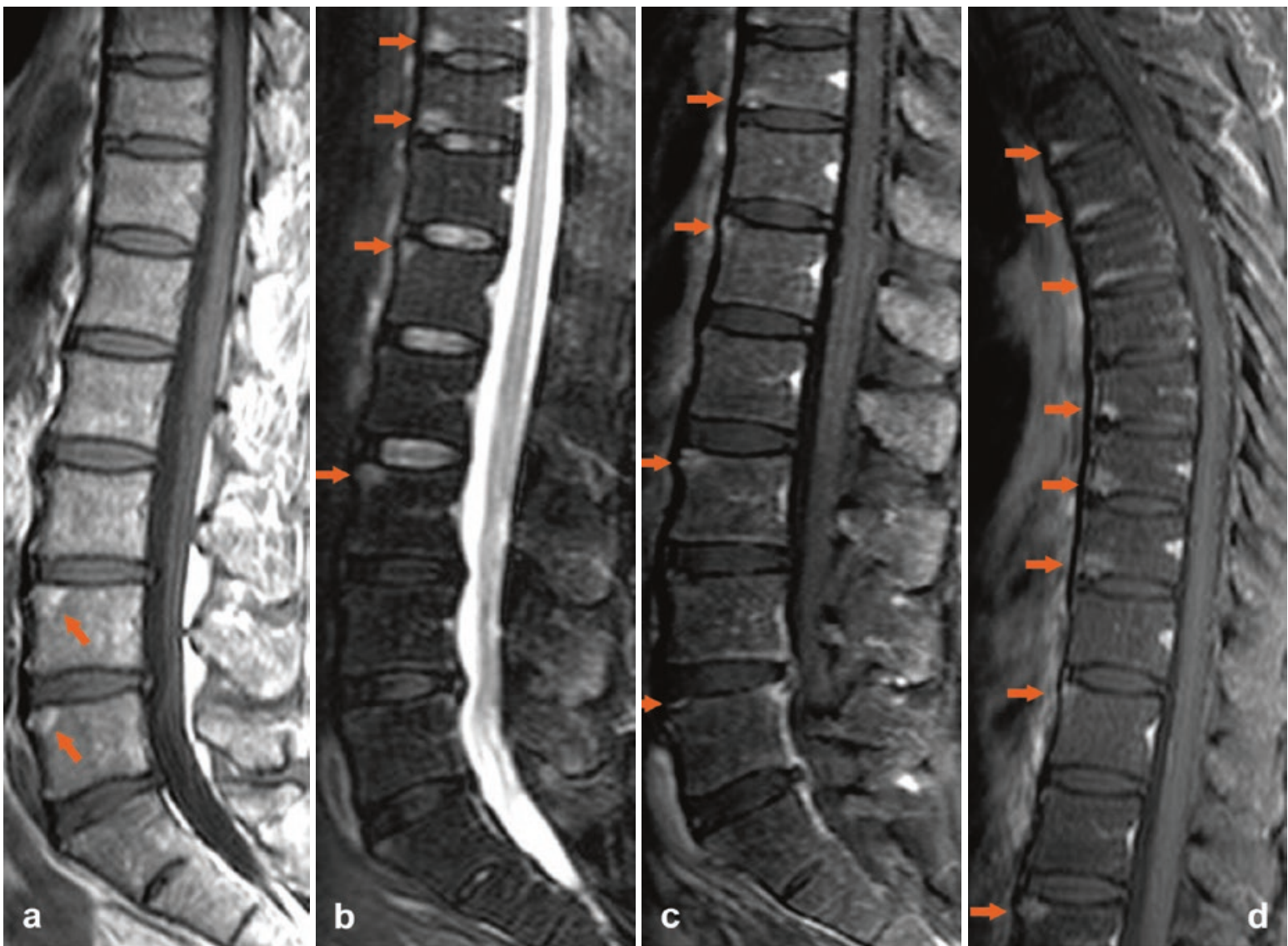

Fig. 1 Sagittal SE T1-weighted image (a), sagittal TSE T2-weighted image with fat saturation (b), sagittal SE T1-weighted images with fat saturation following the administration of contrast medium (c-d). In this patient, we detect multiple foci of predominantly anterior spondylitis. Most of these foci of spondylitis exhibit hyperintense signal in T2

(b, arrows) and contrast enhancement (c, d, arrows), indicating inflammatory activity (in the thoracic and upper lumbar spine); others show postinflammatory areas of fatty degeneration of the bone marrow (anterior edges of the vertebral bodies at L4 and L5, arrows in a). We observe only a single area of posterior spondylitis at L5 